\title{
An 11M-triangles/sec 3D Graphics Clipping Engine for Triangle Primitives
}

\author{
Jaewan Bae, Donghyun Kim, and Lee-Sup Kim \\ Dept. of EECS \\ KAIST \\ Daejeon, Republic of Korea \\ \{jwbae, dhkim\}@mvlsi.kaist.ac.kr, 1skim@ee.kaist.ac.kr
}

\begin{abstract}
This paper describes a clipping engine, which processes the view clipping, perspective division, and view-port mapping of 3D graphics. Complexity of polygon clipping algorithm causes difficulties to implement hardware. We propose a clipping algorithm that shows 3.07 times higher performance than a conventional algorithm. In addition, we propose a scheme to improve performance up to $57 \%$ by eliminating redundant calculations of overlapped vertices without additional hardware. We implement the clipping engine in 0.13um CMOS technology. It processes 11Mtriangles/sec at $166 \mathrm{MHz}$ and has $156 \mathrm{kgates}$ counts.
\end{abstract}

\section{INTRODUCTION}

Graphics acceleration technology has advanced from the field of workstation and PC to consumer electronics. Since applying 3D graphics to consumer electronics restricts hardware cost much more than PC platform, we propose a clipping engine (CEngine) and its clipping algorithm to optimize the high triangle throughput within limited area.

Fig. 1 shows the rendering pipeline to render a primitive in a 3D graphics system [1]. The lighting and transform stages perform nearly identical computation on independent vertices, but gray portion of Fig. 1 performs some calculations on triangle levels. In the remainder, we will describe and discuss aspects of implementing view clipping stage, perspective division stage, and view-port mapping stage of the 3D rendering pipeline.

This paper proposes two features. First, we propose a clipping algorithm specialized to process triangle clipping. Several clipping algorithms for general primitives are studied previously [2], but current 3D graphics acceleration needs a clipping process specialized on triangle. Second, we improve performance by reusing two output vertices at triangle strip. This eliminates redundant calculations of the overlapped vertices at triangle strip. We have implemented CEngine hardware with two features. We analyze the proposed algorithm and measure performance, area and power of the implemented CEngine.

This work is supported in part by the university IT research center program and System IC 2010 program of the government of Korea and by Samsung Electronics

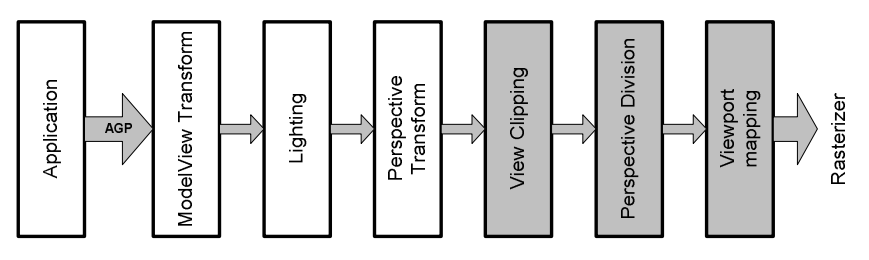

Figure 1. 3D graphics pipeline

The rest of the paper is organized as follows: First, we explain conventional clipping algorithms of line and polygon primitives. We then give an explanation of our clipping algorithm optimized for triangle primitives. Then, we describe the CEngine architecture and hardware implementation. We evaluate our CEngine with simulation results and conclude with an analysis and a summary.

\section{PREVIOUS WORK}

We first review the function of clipping in a rendering system. Clipping is performed to discard portions of the scene that lie outside of the view frustum. The primitive is transformed from model coordinates into perspective coordinates. The view frustum determines which portion of space is visible to viewer. Primitives being outside of the view volume are discarded from further processing stage. Primitives entirely contained in the view volume remain unchanged and are sent directly to the further processing stage, perspective division and view-port mapping stages. Primitives that intersected by the boundary of the view volume are clipped against the view volume, i.e. split primitives into a portion inside and a portion outside of the view volume. Then, the inside portion is then sent to the next processing stage.

One of the line clipping algorithms is Cohen - Sutherland algorithm (CS) [5]. It performs initial tests on a line to determine whether intersection calculations can be avoided. First, endpoint pairs are checked for trivial acceptance. If the line cannot be trivially accepted, region checks are performed as checking intersection endpoint pairs with each 

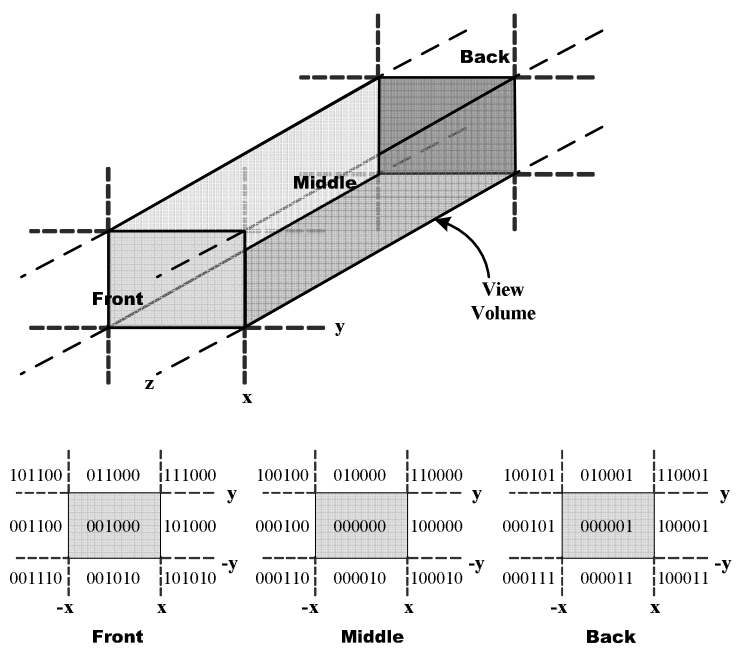

Figure 2. Region outcode in 3D

clip plane of view volume. This algorithm can quickly check whether lines are trivially accepted or not. But because clipping is successively executed against all clip planes, clipping process needs much time.

The Cyrus - Beck algorithm (CB) is based on parametric representation of a line [3]. It classifies the intersections of the line with the boundary edges of the clip rectangle as the Entry and Exit points. The advantage of parametric approach is that it does not compute the intersection points but it calculates the parametric values of intersection points in order to analyze the Entry and Exit points.

The Liang - Barsky algorithms (LB) is a variation of CB algorithm for an early detection of those lines, which may be rejected [4]. Liang and Barsky independently developed a more efficient parametric line-clipping algorithm that is especially fast in the special cases of upright 2D and 3D clip regions. In addition to taking advantage of these simple clip boundaries, it introduced more efficient and exact trivial rejection tests that work for general clip regions. For lines that cannot be trivially rejected by CS algorithm, the rejection tests of the LB are clearly preferable to the repeated clipping required by CS. Calculating two parametric values must be performed for testing whether a triangle is trivially rejected by the LB algorithm. This causes a number of calculations for initial test.

Sutherland and Hodgman's polygon-clipping algorithm uses a divide-and-conquer strategy [2]. The simple problem is to clip a polygon against a single infinite clip edge. Six clip planes in three- dimension, each defining one plane of the clip volume, successively clip a polygon against a clip volume. The polygon clipper clips against six planes in succession. To calculate whether a point lies outside a clip boundary, this tests the sign of the dot product of the normal to the clip boundary and the polygon edge as the LB algorithm does.

\section{PROPOSED TRIANGLE CLIPPING ALGORITHM}

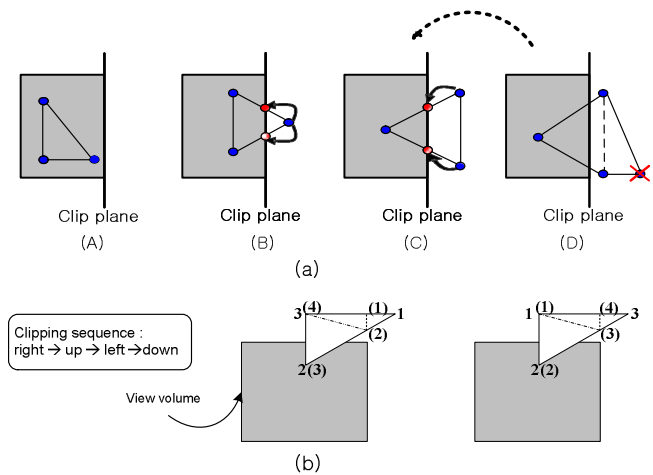

Figure 3. (a) four cases of triangle clipping and (b) exceptions of proposed triangle clipping algorithm

We propose a clipping algorithm optimized triangle primitives. First, outcodes of three vertices of a triangle are calculated by checking the region of each vertex as in Fig. 2 . These outcodes are used to decide whether a triangle is trivially accepted. When a triangle is trivially accepted, this performs perspective division and view-port mapping operation. If not, clipping process is performed. It performs region check against each clip plane. As shown in Fig. 3 (a), this process classifies into four cases against each clip plane. First, three vertices are inside of a clip plane (Case-A). This case doesn't need to perform clipping process against that clip plane. Second, if a vertex is outside, a new vertex is created on that clip plane by clipping process and the existed vertex move from outside of that clip plane to on that clip plane (Case-B). Then, outcodes of the changed vertex and the new vertex is newly calculated. Third, if two vertices are outside, two outside vertices move from outside of clip plane to on that clip plane (Case-C), and outcodes of the two changed vertices are updated. Forth, if three or more vertices are outside, the second vertex among vertices being outside is deleted (Case-D). This case may occur when a new vertex is created by clipping process against a previous clip plane. In succession, above process is repeated against six clip planes. Fig. 3 (b) represents two exceptions of the proposed algorithm. The letter in parenthesis represents the sequence of vertices after clipping by a clip plane. These exceptions may be generated when clipping case against a clip plane is Case-D and first vertex among all vertices is outside against the relevant clip plane. Wrong operation is occurred when the second vertex having to be deleted is not outside against that clip plane. Left figure of Fig. 3 (b) must be deleted the first vertex, and right figure of Fig. 3 (b) must be deleted the forth vertex. The solution is to check the position of second vertex and last vertex against relevant clip plane. The pseudo-code for solving the exceptions is given below.

If second vertex is outside and last vertex is outside Then, first vertex is deleted

Else if second vertex is inside and last vertex is outside Then, last vertex is deleted

Else then, normal clipping process 


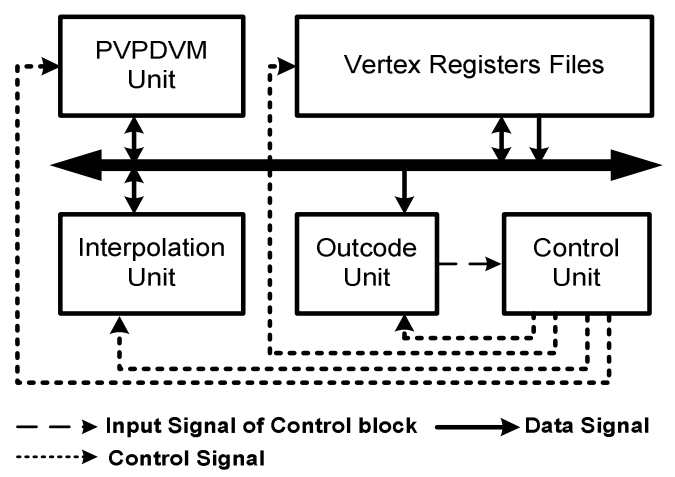

Figure 4. Overall CEngine Architecture

\section{ClipPING ENGINE ARCHITECTURE AND HARDWARE}

Fig. 4 is the overall CEngine architecture. The CEngine consists of five sub-blocks, PVPDVM Unit for calculating parametric value, perspective division and view-port mapping, Interpolation Unit (IU), Outcode Unit (OU), Vertex Register Files (VRF), and Control Unit (CU). VRF outputs the data to PVPDVM Unit and IU, and gets interpolated result from PVPDVM Unit and IU. CU makes signals to generate the control signals of each block. OU sends signals needed to make control signals to CU.

\section{A. Outcode Unit}

Fig. 2 represents outcodes of each region of view volume. Initially, OU is given the position attributes of the three vertices of a triangle from VRF. The outcode calculation of a vertex is easily performed by six FP-comparators. After calculating outcodes of three vertices, it decides whether a given triangle is trivially accepted. If clipping operation is needed, OU makes three signals to control clipping process. The first signal classifies clipping case against each clip plane. It is calculated by summing a group of bit assigned to each clip plane. The second signal represents VRF address of the first outside vertex each clip plane, and the last signal indicates two clipping exceptions.

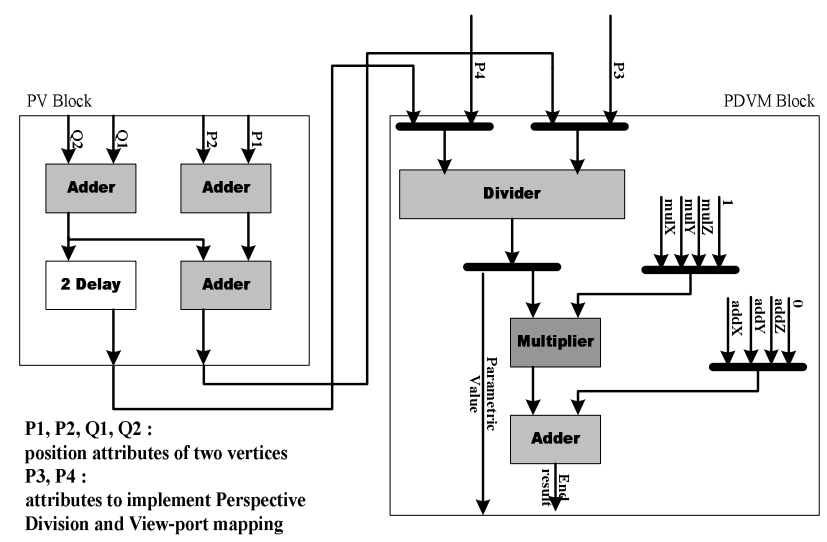

Figure 6. The architecture of PVPDVM Unit

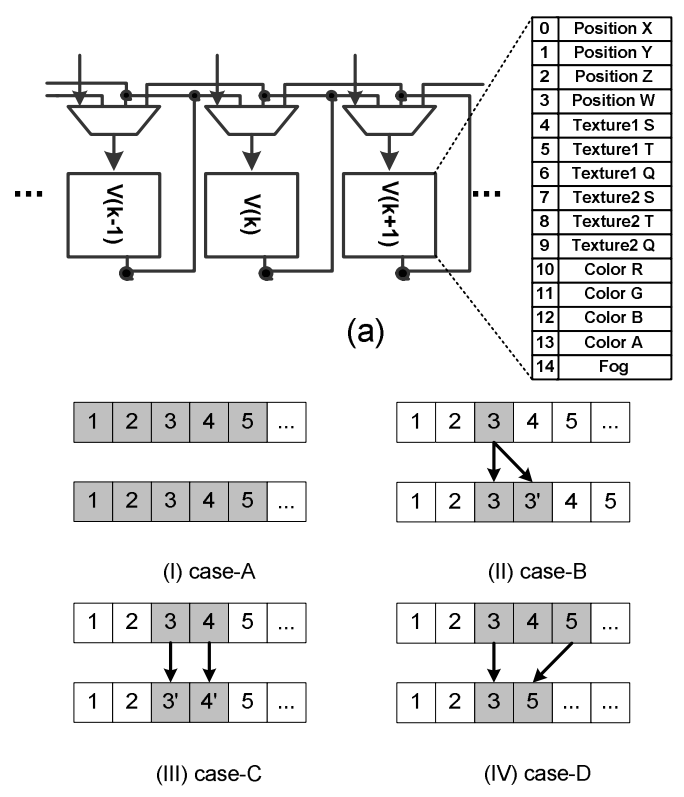

(b)

Figure 5. (a) The VRF structure and attributes of a vertex (b) operations of th VRF corresponding to clipping cases

\section{B. PVPDVM Unit}

PVPDVM Unit performs two operations: First, it calculates parametric value, an intersection point between endpoint pairs and clip planes. Second, it performs the Perspective Division and View-port Mapping operations. As shown in Fig. 6, PVPDVM Unit consists of PV block and PDVM block. PV block don't have a dedicated FP-divider. Instead, FP-divider of PDVM block is shared for calculating parametric value. As receiving coefficients of each position components $(\mathrm{x}, \mathrm{y}, \mathrm{z})$, the PDVM block transform from perspective coordinate to device coordinate for all vertices.

\section{Interpolation Unit}

IU calculates attributes of a new intersected vertex between vertex endpoints by parametric value calculated from PVPDVM Unit. This unit is easily implemented using two FP-adders and a FP-multiplier, as in (1). Where, $P_{0}$ and $P_{1}$ represent attributes of vertex pairs and $t$ indicates parametric value.

$$
P(t)=P_{0}+\left(P_{1}-P_{0}\right) t
$$

\section{Vertex Register Files}

VRF stores input triangle vertices coming from preceding stage of $3 \mathrm{D}$ rendering pipeline. And it output of triangle format for stored vertices to next stage of 3D rendering pipeline. Because six new vertices are able to be created by six clip planes, it has to store the attributes of nine vertices. Fig. 5 (a) shows the VRF structure and attributes of a vertex, and Fig. 5 (b) shows operation of the VRF corresponding to four clipping cases. The VRF architecture supports the shiftfunction from a vertex slot to adjacent vertex slot to cope with four clipping cases. The VRF also easily reuses two of 
TABLE I. THE PROPORTION OF CLIPPED TRIANGLES AT QUAKE3

\begin{tabular}{|c||c|c|c|c|}
\hline & $\begin{array}{c}\text { The proportion of } \\
\text { clipped triangles } \\
\text { to total triangles }\end{array}$ & $\begin{array}{c}\text { The proportion of } \\
\text { clipped triangles by } \\
\text { a plane }\end{array}$ & $\begin{array}{c}\text { The proportion of } \\
\text { clipped triangles by } \\
\text { two plane }\end{array}$ & $\begin{array}{c}\text { The proportion of } \\
\text { clipped triangles by } \\
\text { over three plane }\end{array}$ \\
\hline SH Algorithm & $8.16 \%$ & $6.395 \%$ & $1.693 \%$ & $0.07 \%$ \\
& $(100 \%)$ & $(78.37 \%)$ & $(20.76 \%)$ & $0.7 \% \%)$ \\
\hline Proposed triangle & $10.817 \%$ & $8.782 \%$ & $1.962 \%$ & $0.0724 \%$ \\
Aglorithm & $(100 \%)$ & $(81.187 \%)$ & $(18.138 \%)$ & $(0.67 \%)$ \\
\hline 0: the proportion about total clipped triangles
\end{tabular}

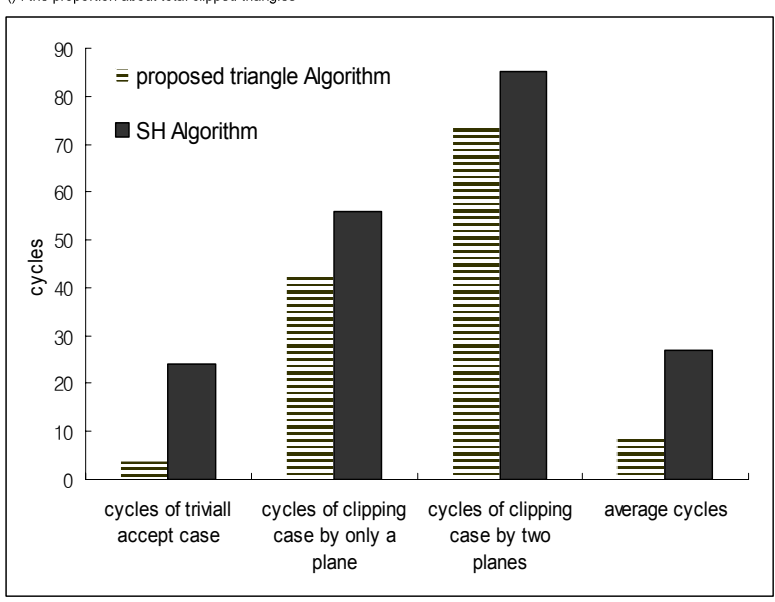

Figure 7. comparison proposed algorithm with $\mathrm{SH}$ algorithms against cases of acceptance trivially, clipping by only a plane and clipping by two plane without texture

output vertices in triangle strip mode. If both successive triangles are trivially accepted in triangle strip mode, second and third vertices of the prior triangle processed by perspective division and view-port mapping operation are stored in first and second position of VRF by shift operation. Then, the VRF only gets third vertex of next triangle, because perspective division and view-port mapping operation of first and second vertices of next triangle is already performed in prior triangle. For the only last vertex, it performs perspective division and view-port mapping operation. This operation improves performance as reducing redundant calculations without additional registers for reusing.

\section{DISCUSSION}

Scenes of QUAKE3 are used in the experiment and analysis of the proposed CEngine implemented in VerilogHDL RTL. The time exhausted by polygon processing in the clipping stage depends on the number of clip planes intersected with a triangle. Hence, we survey the proportion of clipped triangles to total triangles at several scenes and the number of clip plane affecting to a triangle as clipping process. Table 1 shows the proportion of clipped triangles to total triangles at scenes of QUAKE-3 and the proportion of the triangles affected by a clip plane, two clip planes, and three or more clip planes at scenes. Table 1 represents that the proposed algorithm has more clipping case than $\mathrm{SH}$ algorithm since initial test of the proposed algorithm is fast but inexact. As based on the data of Table 1, Fig. 7 (b) shows simulated clock cycle time of the proposed algorithm compared with $\mathrm{SH}$ algorithm. The result shows the proposed
TABLE II. SYNTHESIZED RESULT OF CENGINE

\begin{tabular}{|c|c|c|}
\hline & Area(gates) & Power(mW) \\
\hline Outcode Unit & 5053 & 0.463 \\
\hline PVPDVM Unit & 41386 & 7.525 \\
\hline Interpolation Unit & 14980 & 2.09 \\
\hline $\begin{array}{c}\text { Vertex Registers } \\
\text { Files }\end{array}$ & 82643 & 27.1 \\
\hline Control Unit & 6854 & 4.75 \\
\hline Total & 155886 & 41.97 \\
\hline
\end{tabular}

algorithm has 3.07 times performance improvement than $\mathrm{SH}$ algorithm for processing a triangle. This is why $\mathrm{SH}$ algorithm spends too much time to decide whether a triangle is trivially accepted, but most of the total triangles are trivially accepted.

Reducing redundant calculations by reusing the two outputted vertices improves the performance up to $57 \%$. It is reasonable why most of the scenes in current graphics models consist of triangle strips.

We reduce hardware cost of CEngine as eliminating a dedicated FP-divider for calculating parametric value. In addition, the VRF structure without additional registers to reuse overlapped vertices contributes to reduce the area of CEngine.

The proposed CEngine is implemented in 0.13um CMOS technology and provides the performance up to $11 \mathrm{Mtriangles} / \mathrm{sec}$ at $166 \mathrm{MHz}$. The gate counts and average power are shown in Table 2. We apply the proposed CEngine to the embedded 3D graphics SOC system.

\section{CONCLUSION}

This paper proposes an algorithm specialized to process triangle clipping and a scheme to eliminate redundant calculations of the overlapped vertices. These features improve the performance within a limited area. The proposed CEngine processes the triangles with up to $11 \mathrm{M}$-triangles/sec, and it has the power consumption of $42 \mathrm{~mW}$ at $166 \mathrm{MHz}$ and the area of 156kgates.

\section{REFERENCES}

[1] Bengt-Olaf Schneider, "Efficient Polygon Clipping for an SIMD Graphics Pipeline", IEEE Transactions On Visualization and Computer Graphics, vol.4, no.3, pp. 272-285, July-September 1998.

[2] Suland, I.E., and Hodgman, G.W., "Reentrant Polygon Clipping," Communication of ACM, vol.17, pp. 32-42, January 1974.

[3] Cyrus, M. and J. Buck, "Generalized two- and three dimensional clipping," Computers and Graphics, pp. 23-28, 1978.

[4] Liang, Y-D., and Barsky, B., "A New Concept and Method for Line Clipping," ACM Transactions on Graphics, vol.11, pp. 276-290, January 1984.

[5] Foley, van Dam, Feiner, and Hughes, "Computer Graphics : Principles and Practice" $2^{\text {nd }}$ edition in C, Wesley, pp. 113-117. 